\title{
THE IMPORTATION OF NEW PLANT GERMPLASM
}

\author{
G.R.G. CLOVER, L.W.M. LIEFTING, L.I. WARD, B.D. QUINN, \\ H.L. THOMPSON and T. WEI
}

\begin{abstract}
Plant Health and Environment Laboratory, Investigation and Diagnostic Centre, Biosecurity New Zealand, Ministry of Agriculture and Forestry, PO Box 2095, Auckland, New Zealand

Corresponding author: cloverg@maf.govt.nz
\end{abstract}

Agriculture in New Zealand is dependent on introduced plants. Access to new genetic material is crucial to enable the sector to remain competitive by introducing traits such as disease resistance and developing improved varieties.

All plants imported into New Zealand must meet certain requirements to ensure that damaging pests and diseases are not introduced. The import requirements are determined by the Ministry of Agriculture and Forestry (MAF) on the basis of risk analyses and published as import health standards. Requirements include inspection, testing or treatment of the plants, prior to export, upon arrival at the New Zealand border and/or in post-entry quarantine (PEQ).

Depending on the importance of the crop and the severity of the potentially associated pests and diseases, imported plants may be released at the border (e.g. seed of most arable crops and ornamental plants) or following PEQ. Unless sourced from MAF-approved high-health schemes, many important crops (e.g. apple budwood, hop rhizomes, potato tissue culture and strawberry plants) must be imported into the most contained level of PEQ (Level 3). Plants from high-health facilities overseas may be imported with less onerous requirements such as reduced testing and/or a shorter quarantine period.

Level 3 PEQ facilities in New Zealand are privately run and two companies (Linnaeus and HortResearch) provide facilities for crops such as avocados, grapevines and kiwifruit. Plants in Level 3 PEQ must undergo testing prior to clearance and therefore facilities must have access to a testing laboratory. Private companies only provide testing for a limited number of crops and therefore MAF's Plant Health and Environment Laboratory (PHEL) has been mandated to:

- develop and validate PEQ testing manuals (describing the materials and methods used to test plants in quarantine) and

- provide PEQ testing (on a cost-recovered basis) that is not offered by private industry.

PHEL is conducting research to enable germplasm to be imported more cheaply, for example by developing generic tests that detect a range of pests (e.g. a virus genus). The laboratory has also reviewed the pests associated with pollen, and is collaborating with other researchers to develop detection methods to test imported pollen directly. 